\title{
Measurement of Linear Attenuation Coefficient of Tc-99m using Planar Gamma Camera Image
}

\author{
Md. Nahid Hossain, Kamila Afroj Quadir, Md. Nurul Islam, Tanvir Ahmed Biman, Ferdoushi Begum \\ National Institute of Nuclear Medicine and Allied Sciences, Bangladesh Atomic Energy Commission, Dhaka
}

Address for Correspondence : Md. Nahid Hossain, Senior Scientific Officer, National Institute of Nuclear Medicine and Allied Sciences, Bangladesh Atomic Energy Commission, BSM Medical University, Dhaka, Bangladesh. Email: nahidhssn@yahoo.com

\begin{abstract}
Introduction: Attenuation is one of the most important factors which have an impact on the accuracy on quantitation of activity when using gamma camera image in a medium. Due to attenuation a number of photons are lost and there is also loss of counts which otherwise would have been included in the images. The purpose of this study is to estimate the linear attenuation coefficient for Tc-99m with water filled phantom using conjugate view method and transmission method and compare the obtained measurements with the reference values.

Materials and Methods: To determine the linear attenuation coefficient of radionuclide Tc-99m using conjugate view method, the sensitivity of the gamma camera was determined. $30 \mathrm{MBq}$ of Tc-99m was taken which was measured in dose calibrator and gamma camera images were acquired. Then the sensitivity was calculated as CPS/MBq. A Jaszczak phantom was filled up with water and a small sized cylindrical container filled with activity of $30 \mathrm{MBq}$ Tc-99m liquid was placed inside the Jaszczak phantom centrally. The phantom was scanned on a dual head gamma camera and the images were taken and counts from the images were estimated. After estimation of all values, the linear attenuation coefficient was calculated using conjugate view formula. The linear attenuation coefficient was also measured from the transmission imaging. The transmission factor (TF) was determined by measuring the ratio of count rates obtained with Tc-99m under gamma camera, with and without the phantom. Results: The linear attenuation coefficient was calculated $0.1467 \pm 0.0057$ from conjugate view method and $0.1483 \pm 0.0023$ from transmission measurements. Both results were found within $\pm 5 \%$ of the established value $\left(0.15 \mathrm{~cm}^{-1}\right)$. Conclusions: Correction for attenuation is required for the accurate estimation of activity. For conjugate view method, the attenuation coefficient was measured with a known activity of Tc-99m. The attenuation coefficient was also estimated by transmission measurements. The accuracy of the activity measurement is highly dependent on the accuracy of the attenuation coefficient used in the calculation.
\end{abstract}

\section{INTRODUCTION}

A number of factors impact the accuracy of activity quantitation in gamma camera imaging. The most important factor is attenuation in the medium containing the activity. Attenuation removes photons by means of the loss of counts which otherwise would have been included in the images (1). The linear attenuation coefficient $\left(\mu_{\mathrm{e}}\right)$ describes the fraction of gamma rays that is absorbed or scattered per unit 
thickness of the absorber. The unit of linear attenuation coefficient is $\mathrm{cm}^{-1}$. The linear attenuation coefficient mainly depends upon the composition of the attenuating material and the photon energy (2). When gamma photons are attenuated, the number of photons that travel through the material is given by the following equation:

$$
\mathbf{I}=\mathbf{I}_{\mathbf{0}} \mathbf{e}^{-\mu d}
$$

Where $\mathrm{I}_{0}$ and I represent counts detected without attenuation and with attenuation respectively through the material thickness $d$, with attenuation coefficient $\mu$. For Technetium-99m which contains $140 \mathrm{keV}$ gamma energy, the $\mu$ value is $0.15 / \mathrm{cm}$ for most of human tissues; but higher for higher density bone and less for lung, which contains air (3).

The purpose of this study is to estimate the linear attenuation coefficient of Tc-99m with water filled phantom using methods mentioned above and compare to the reference values. The reference value of the linear attenuation coefficient for Tc-99m is $0.15 / \mathrm{cm}$ for water, which is almost same as for the human tissues value (4).

\section{MATERIALS AND METHODS}

Conjugate view method for gamma camera planar images was used to determine the linear attenuation coefficient for radionuclide Tc-99m in water. To determine the sensitivity of the gamma camera, 30 $\mathrm{MBq}$ of Tc-99m was taken which was measured from a dose calibrator (Model Veenstra, VDC-405, Netherlands). A $10 \mathrm{~cm}$ diameter and $3 \mathrm{~mm}$ thick source containing $30 \mathrm{MBq}$ of Tc-99m was placed 10 $\mathrm{cm}$ from the collimator surface and a dual head gamma camera (Siemens e.cam series) acquired images for a fixed time $(600 \mathrm{sec})$. The number of counts per second was determined from region of interest (ROI) in the image with correction of background activity. The sensitivity, also known as system calibration factor of camera, was calculated as CPS/MBq. The low energy all purpose (LEAP) collimator was employed for all imaging because of the low energy of Tc-99m (140 KeV). A Jaszczak phantom was taken without its inserts and filled up with water. A smaller sized cylindrical container with activity of $30 \mathrm{MBq}$ Tc-99m liquid with water placed inside the Jaszczak phantom centrally which emitted gamma rays where the photons were attenuated by the water in the phantom (Figure 1). The length of the cylindrical container was $5.2 \mathrm{~cm}$ and thickness or diameter was $3 \mathrm{~cm}$. The phantom scanned on the dual head gamma camera and the images were taken both anteriorly-posteriorly and the counts from the images were estimated. The length and thicknesses of the phantom were $20 \mathrm{~cm}$ and 22 $\mathrm{cm}$. 


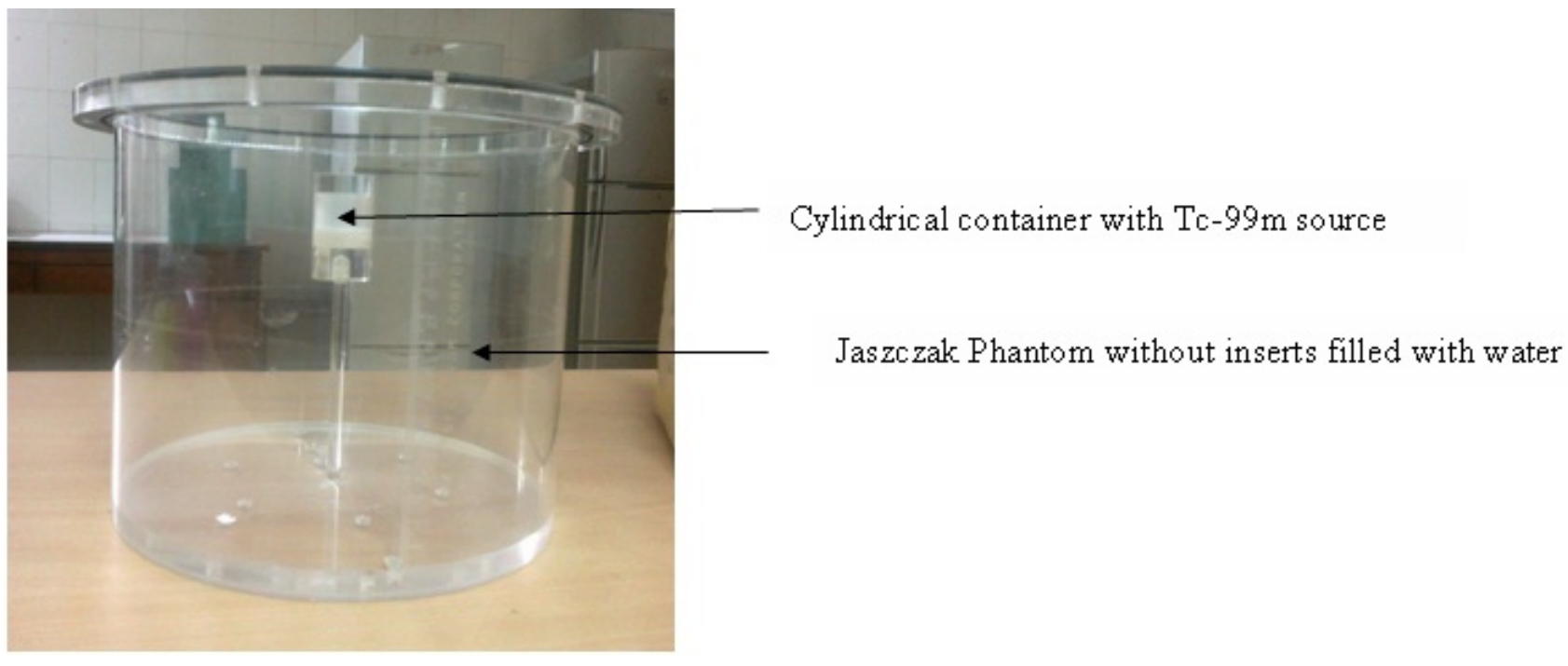

Figure 1: Cylindrical container with Tc-99m source placed centrally in water filled Jaszczak Phantom

The background was also corrected. For Background correction, Regions of interest (ROI) were drawn around the Tc-99m source on the gamma camera images in the anterior and posterior views. The same size of a background region as the source was defined adjacent to the source ROI and background corrections were carried out from the data (5). The methods we used here was the conventional background correction method in which the net counts in the region, D was defined as.

$$
\mathrm{D}=\mathrm{D}^{\prime}-\mathrm{D}_{\mathrm{B}}
$$

Where $D^{\prime}=$ Gross counts in source region, $D_{B}=$ Counts in the background region (accounting for number of pixel in the regions).

The other method that was used is known as the source volume background correction method as it considers the source thickness. This method of background correction is also known as Buijs method (6). This method requires a correction factor for the source volume.

$$
\mathrm{D}=\mathrm{D}^{\prime}-\mathrm{D}_{\mathrm{B}} * \mathrm{~F}
$$

Where $\mathrm{F}=1-l / \mathrm{L}, l=$ source thickness, $\mathrm{L}=$ phantom thickness.

After estimation of all values, the linear attenuation coefficient was calculated using conjugate view formula. The calculation from conjugate view formula using MIRD pamphlet no.16 is (7).

$$
A=\sqrt{\frac{I_{A} I_{P}}{\tau}} \times \frac{f}{C}
$$




$$
\text { Therfore } \quad \tau=I_{A} I_{P} \cdot \frac{f^{2}}{A^{2} C^{2}}
$$

Where, $f$ provides the correction for the source region attenuation. $f$ is a correction dependent on object or source thickness that is always less than or equal to 1 and is 1 for small objects (8). $I_{A}$ and $I_{P}$ determine the anterior and posterior conjugate view count rates for the region to be quantitated through ROI analysis, $\tau$ is the transmission factor and $\mathrm{C}$ is the system calibration factor (count rate per unit activity). After finding all values, we calculated the $\tau$ value (transmission factor) from equation (3).

The transmission factor is:

$$
\begin{aligned}
\tau & =e^{-\mu_{e} L} \\
\text { Therfore } \quad \mu_{e} & =-\frac{\ln \tau}{L}
\end{aligned}
$$

The other method for determining the linear attenuation coefficient value was measured from the transmission imaging. The transmission factor (TF) represents across the phantom thickness through the region of interest with linear attenuation coefficient. A transmission scan was performed. The water filled Jaszczak phantom scanned along with the flood source filled with water which was mixed with $20 \mathrm{mCi}$ of Tc-99m. A blank scan of flood source without the phantom was also performed using the same acquisition parameters of camera. The transmission factor was determined by measuring the ratio of count rates, obtained by using the Tc-99m both with and without the phantom in position using equation (5).

$$
\mathrm{TF}(\tau)=\text { cts/pixel }(\text { transmission }) / \mathrm{cts} / \text { pixel }(\text { blank image }) \ldots \ldots .
$$

After calculating the transmission factor, we measured the linear attenuation coefficient value from equation (4)

\section{RESULTS}

The linear attenuation coefficient was calculated with measured system calibration factor, background correction factor, known activity and count rates using conjugate view formula. From conjugate view method calculation, the $\mathrm{L}$ value for Phantom was $22 \mathrm{~cm}$ which was the diameter of the phantom, $\mathrm{f}$ value was 1 for very small source, A was $30 \mathrm{MBq}$; the known activity and $\mathrm{C}$ value was $148 \mathrm{CPS} / \mathrm{MBq}$. 
Table 1: $\mu_{\mathrm{e}}$ calculation from conjugate value formula

\begin{tabular}{|c|c|c|c|c|}
\hline $\mathrm{I}_{\mathrm{A}}$ & $\mathrm{I}_{\mathrm{P}}$ & $\tau$ & $\mu_{\mathrm{e}}$ & Mean $\mu_{\mathrm{e}}$ \\
\hline 985 & 827 & 0.0413 & 0.145 & \multirow{2}{*}{$0.1467 \pm 0.0057$} \\
\cline { 1 - 4 } 889 & 765 & 0.0345 & 0.153 & \\
\hline 1004 & 867 & 0.0442 & 0.142 & \\
\hline
\end{tabular}

Table 1: $\mu_{\mathrm{e}}$ calculation from transmission measurement

\begin{tabular}{|c|c|c|c|c|}
\hline $\begin{array}{c}\text { Transmission } \\
\text { Counts/pixel }\end{array}$ & $\begin{array}{c}\text { Blank } \\
\text { Counts/pixel }\end{array}$ & $\tau$ & $\mu_{\mathrm{e}}$ & $\mu_{\mathrm{e}}$ Mean \\
\hline 20.29 & 517.27 & 0.0392 & 0.147 & \\
\hline 21.35 & 596.29 & 0.0358 & 0.151 & $0.1483 \pm 0.0023$ \\
\hline 20.97 & 531.57 & 0.0395 & 0.147 & \\
\hline
\end{tabular}

From second method, the linear attenuation coefficient was calculated from transmission factor formula using transmission imaging. Again the L value for Phantom was $22 \mathrm{~cm}$ (the diameter of the phantom). For the conjugate view method, the average of 5 readings resulted in linear attenuation coefficient value of $0.1467 \pm 0.0057 \mathrm{~cm}^{-1}$. From second method, the average linear attenuation coefficient value was found to be $0.1483 \pm 0.0023 \mathrm{~cm}^{-1}$. Both results were found within $\pm 5 \%$ of the established value $\left(0.15 \mathrm{~cm}^{-1}\right)$.

\section{DISCUSSIONS}

From the conjugate view method, the attenuation coefficient was measured with a known activity of Tc-99m. In the second method, the attenuation coefficient was estimated by transmission measurements using the same camera, collimators and camera acquisition parameters throughout the study. The measurement of attenuation coefficient from gamma camera imaging was better when this was determined by transmission measurements. The background correction method, which considers the source volume combined with quantitative planar imaging; provides more accurate results for the estimation of attenuation coefficient value, compared with the conventional method without background subtraction. 


\section{CONCLUSIONS}

Correction for attenuation is required for the accurate estimation of activity. The measurement of attenuation coefficient for different gamma energy photon with different organs is very important to determine the exact attenuation by the organs. The exactness of attenuation correction becomes very essential in cases where patient specific image based dosimetry is being performed. The accuracy of the activity measurement is highly dependent on the accuracy of the attenuation coefficient used in the calculation.

\section{REFERENCES}

1. Michael King, Ph.D., and Troy Farncombe, Ph.D. An Overview of Attenuation and Scatter Correction of Planar and SPECT Data for Dosimetry Studies. Cancer Biother Radiopharm. 2003 Apr;18(2):181-90.

2. Saxby Browna, Dale L. Bailey, Kathy Willowson, Clive Baldock. Investigation of the relationship between linear attenuation coefficients and CT Hounsfield units using radionuclides for SPECT. Science Direct Applied Radiation and Isotopes 66 (2008) 1206-1212.

3. Heather E. Patterson, Brian F. Hutton. Distance Assisted Training Programme for Nuclear Medicine Professionals; SPECT and SPECT/CT; Module 13 Unit 24; P32.

4. Takashi Ichihara, Koichi Ogawa, Nobutoku Motomura, Atushi kubo and Shozo Hashimoto. Compton scatter compensation using the triple-energy window method for single and dual isotope SPECT. The Journal of Nuclear Medicine; Vol. 34. No. 12; December 1993, P22162221.

5. Kristina Norrgren Ph.D., Sigrid Leide Svegborn Ph.D., Johan Areberg Ph.D., Sören Mattsson Ph.D.; Accuracy of the Quantification of Organ Activity from Planar Gamma Camera Images.; Cancer Biotherapy \& Radiopharmaceuticals; Volume 18, Number 1, 2003; 125-131.

6. Buijs WCAM, Siegel JA, Corstens FHM. Estimation of absolute organ activity using five different methods of background correction: a phantom study [Abstract]. Eur J Nucl Med 1997;24:931.

7. Siegel JA, Thomas SR, Stubbs JB, Stabin MG, Hays MT, Koral KF, et al. MIRD pamphlet No. 16: Techniques for quantitative radiopharmaceutical biodistribution data acquisition and analysis for use in human radiation dose estimates. The Journal of Nuclear Medicine; Vol. 40. No. 2 (suppl); February 1999, 37S-61S.

8. K.F koral, H. Zaidi. Methods for Planar image Quantification. Quantitative analysis in nuclear medicine imaging. Springer 2006; P414-434. 